\title{
Disección de arteria carótida interna secundario a accidente en motocicleta
}

\section{Internal carotid artery dissection secondary to motorcycle accident}

\author{
Enrique Villarreal García1, José Raymundo Medina Romero², Eduardo Díaz Juárez², \\ Leonel Ramírez Abrego'
}

\author{
${ }^{1}$ Residencia de Neurocirugía, Servicio de Neurocirugía, Hospital General 450 Durango, Secretaría de Salud, Durango. Durango, \\ México. \\ ${ }^{2}$ Servicio de Neurocirugía, Hospital General 450 Durango, Secretaría de Salud, Durango. Durango, México.
}

\section{Resumen}

La disección traumática de la arteria carótida interna (DTACI) puede ser secundaria a causas iatrogénicas, desórdenes del tejido conectivo y traumatismos que ocasionan la ruptura de la íntima ya sea por contusión directa o diversos grados de rotación e hiperextensión del cuello. El caso clínico corresponde a paciente masculino de 20 años edad el cual sufre accidente en motocicleta, a su ingreso a hospitalización la evaluación inicial del paciente es estable, sin déficit neurológico. A las 9 horas disminución del Glasgow, tumefacción progresiva en cuello lado derecho y dolor intenso; se realizó angiotomografía con sangrado en nacimiento de paquete vascular derecho y disminución de la de la luz de arteria carótida interna ipsilateral. Se sometió a procedimiento quirúrgico para exploración vascular de cuello y corrección de laceración de vena yugular interna. Se inicia tratamiento antiplaquetario. A las 24 horas posteriores presenta datos clínicos e imagenológicos de evento vascular cerebral isquémico, que requirió craniectomía descompresiva. A las 48 horas, infarto bien establecido de territorio de arteria cerebral media derecha secundario a embolismo por disección carotidea derecha sobre su bifurcación. A los 7 días se egresa con tratamiento antiplaquetario a largo plazo.

Palabras clave: Disección, arteria carótida interna, embolismo.

\section{Abstract}

The traumatic dissection of the internal carotid artery can be secondary to iatrogenic, connective tissue disorders and less commonto trauma that causes the rupture of the intima either by direct contusion or various degrees of rotation or hyperextension of the neck. The clinical case corresponds to a 20 -year-old male patient who suffers a motorcycle accident. Upon admission to hospitalization, the initial evaluation of the patient is stable, without neurological deficit. After 9 hours decrease in Glasgow, progressive swelling in the right-side neck and severe pain; Anangiotomography was performed with bleeding from the right vascular bundle and a decrease in the lumen of the ipsilateral internal carotid artery. A surgical procedure was performed for vascular exploration of the neck and correction of internal jugular vein laceration. Antiplatelet therapy is started. After 24 hours, he presented clinical and imaging data of an ischemic cerebrovascular event, wich required a decompressive craniectomy. After 48hours, a well-established infarction of the right middle cerebral artery territory secondary to embolism due to right carotid dissection on its bifurcation. After 7 days, he was discharged with long-term antiplatelet treatment.

Key words: Dissection, internal carotid artery, embolism.

\section{Correspondencia a:}

Enrique Villarreal García

Dirección Postal, Bulevar José María Patoni S/N, CP 34206, Durango.

Durango México.

Teléfono, 6181373328.

kikevg90_05@hotmail.com 


\section{Introducción}

La disección arterial cérvico cerebral (DACC) representa el $2 \%$ de los infartos cerebrales, siendo más frecuente dentro de población joven, la cual se divide en disección del sistema carotideo cervical y cerebral; y del sistema vertebro basilar. La disección cervical de la arteria carótida interna $(\mathrm{ACl})$ representa entre el $80 \%$ y $90 \%$ de todas las disecciones cervicocerebrales, se reporta una de incidencia 3,5 por 100.000 habitantes en mayores a 20 años; sin embargo, se encuentra infra diagnosticada ${ }^{1,2,3,4,5}$.

La tasa de morbimortalidad en esta patología es del 30\%, y las lesiones neurológicas secuelares $80 \%^{6}$; constituye el $20 \%$ de los casos de infarto cerebral en adultos jóvenes ${ }^{7}$.

Esta patología contempla la ruptura de la íntima con desarrollo de hematoma intramural dentro de las capas de la túnica media, se caracteriza por tener un crecimiento excéntrico en dos modalidades, disección subintimal asociada a estenosis luminal y disección subadventicia asociada a dilatación arterial5 ${ }^{5,8,9}$

La etiología incluye eventos iatrogénicos, desórdenes del tejido conectivo, o traumatismos, ya sea contusión directa con hiperextensión o rotación forzada del cuello ${ }^{10,11}$.

La disección cervical de $\mathrm{ACl}$ se localiza con mayor frecuencia a 2 centímetros distal a la bifurcación carotidea (c2-c3) ${ }^{12}$.

Clínicamente, el $50 \%$ de las DTACI son asintomáticas en las primeras 12 horas y representan la progresión de la disección hacia trombosis o embolización ${ }^{12}$.

Se puede encontrar dolor ipsilateral de cabeza y cuello, síndrome de Horner por lesión del plexo pericarotideo o parálisis de nervios craneales bajos; el déficit neurológico es inconsistente con el hallazgo de la tomografía axial computarizada. Los datos de isquemia se presentan entre el $50 \%$ y $95 \%$ de los casos, pueden existir infartos territoriales o ataques isquémicos transitorios $(50 \% \text { a } 75 \%)^{13,14}$.

La disección cervical de la $\mathrm{ACl}$ contempla cinco categorías, grado I cuando la oclusión de la luz arterial es menor o igual al $25 \%$, grado II cuando la oclusión supera el $25 \%$ de la luz arterial, grado III cuando se constituye como pseudoaneurisma, grado IV cuando existe oclusión arterial, y el grado $\checkmark$ contempla la sección arterial $\left.\right|^{5,15,16}$.

El manejo es conservador, contempla terapia antitrombolítica, anticoagulante o antiplaquetaria durante 3 a 6 me$\operatorname{ses}^{5,10,17}$. No se encuentra diferencia significativa en la elección del manejo conservador; en algunos casos tratamiento endovascular o quirúrgico cuando existe el acceso al sitio lesionado y se cuenta con equipo médico adecuado ${ }^{1}$.

La embolización en las arterias cerebrales fue encontrada en $36 \%$ de disección de $\mathrm{ACl}$ así como eventos isquémicos transitorios en el $50 \%$ de los pacientes ${ }^{18}$.

\section{Caso clínico}

Paciente atendido en diciembre de 2018 en el Hospital General de Durango, México de la Secretaría Salud.

1.- Antecedentes. Masculino de 20 años con dominancia diestra, alcoholismo, tabaquismo y consumo de marihuana ocasional, plastia inguinal derecha hace 15 años.
2.- Inicio. 20 minutos antes del ingreso al servicio de urgencias, sufre accidente en motocicleta sin uso de casco, tipo choque frontal contra automóvil, siendo expulsado de su vehículo e impactándose el cráneo sobre el parabrisas, sin pérdida del estado de alerta.

a) Exploración física. General, signos vitales normales. Cráneofacial, múltiples contusiones y escoriaciones en cara, edema palpebral derecho con oclusión de órbita, oclusión dental limitada por desviación lateral derecha de la mandíbula, asimetría facial. Cuello, cilíndrico, tráquea central, no deformidades, pulsos presente, no soplos. Cardiopulmonar y abdomen, sin alteraciones. Extremidades, dolor intenso a la palpación en tercio distal de antebrazo derecho.

b) Exploración neurológica. Escala de coma de Glasgow (ECG) 15 puntos (v4, o5, m6), lenguaje limitado por lesión de mandíbula, juicio, praxis, y gnosis sin alteración. Pares craneales, sin alteración. Extremidades, fuerza 5/5, sensibilidad conservada, reflejos osteotendinosos $++/++++$, babinski y sucedáneos negativos. Cerebelo, eumetría, diadococinesia, ausencia de nistagmos. Meníngeos, Kernig y Brudzinski negativos.

c) Exploración Imagenológica. Radiografía, cuello, tórax y abdomen sin alteración, fractura de cubito distal derecho. TAC cráneo simple, sin hallazgos de relevancia.

d) Diagnóstico.Traumatismo cráneo encefálico leve por ECG, fractura mandibular LeFort II, fractura de cubito distal derecho.

3.- Evolución ( 9 horas del inicio). Deterioro del estado de alerta ECG 7 pts $\left(\mathrm{O}_{2}, \mathrm{~V} 1, \mathrm{M} 4\right)$. Presencia de tumefacción cervical derecha $4 \times 5$ centímetros en triángulo anterior del cuello, pulsátil, irregular, consistencia blanda, bordes mal definidos; pupilas anisocóricas dependiente de pupila derecha $5 \mathrm{~mm}$, hiporreflécticas, reflejos oculocefálicos presentes; extremidades izquierdas con hipoestesia, no existe retiro al estímulo doloroso, reflejos osteotendinosos $+++/++++$, Babinski izquierdo presente.

a) TAC cráneo simple. Relación sustancia gris sustancia blanca perdida con pérdida de surcos, con obliteración de ventrículos laterales por edema de parénquima cerebral, cisternas perimecencefálicas se encuentran parcialmente cerradas, tallo cerebral se encuentra sin cambios de densidad (Figura 1).

b) AngioTAC. Reconstrucción en tercera dimensión y sustracción ósea en la cual se observa de rostral a caudal porción proximal de cuello zona de sangrado en nacimiento de paquete vascular derecho con desplazamiento hacia lateral de vena yugular interna ipsilateral, presencia de trayecto de arteria carótida común derecha con bifurcación y disminución de la luz de arteria carótida interna a 2 centímetros de la bifurcación carotidea en porción extracraneal, con ausencia de paso de material de contraste (Figura 1).

c) Diagnóstico. Síndrome corticoespinal completo para el hemisferio derecho, síndrome de herniación uncal, laceración de vena yugular interna y disección carotidea derecha.

d) Manejo General. Se inicia manejo avanzado de vía aérea y sedación, se inició antiagregación plaquetaria con 200 mg acido acetil salicílico.

e) Exploración quirúrgica. Trauma vascular de cuello zona II, hematoma no evolutivo de $70 \mathrm{~mm}$ y laceración y reparación de vena yugular interna de 2 centímetros. 


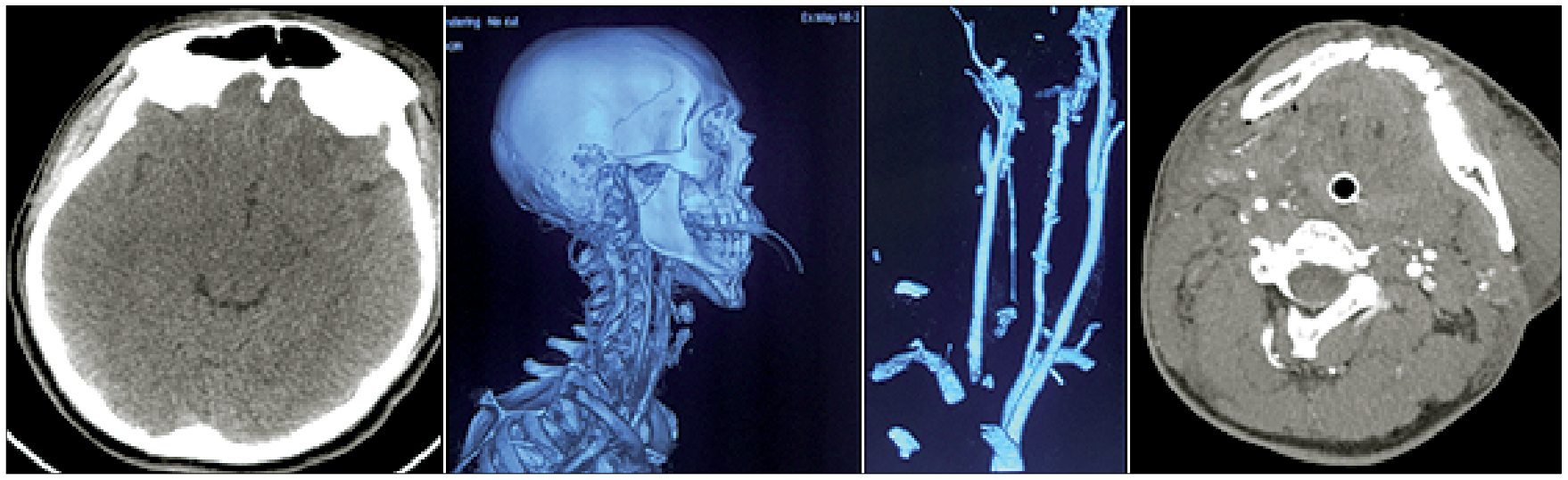

Figura 1. Tomografía axial computarizada simple de cráneo y angiografía con tomografía axial computarizada con reconstrucción en tercera dimensión a las 9 horas de evolución.
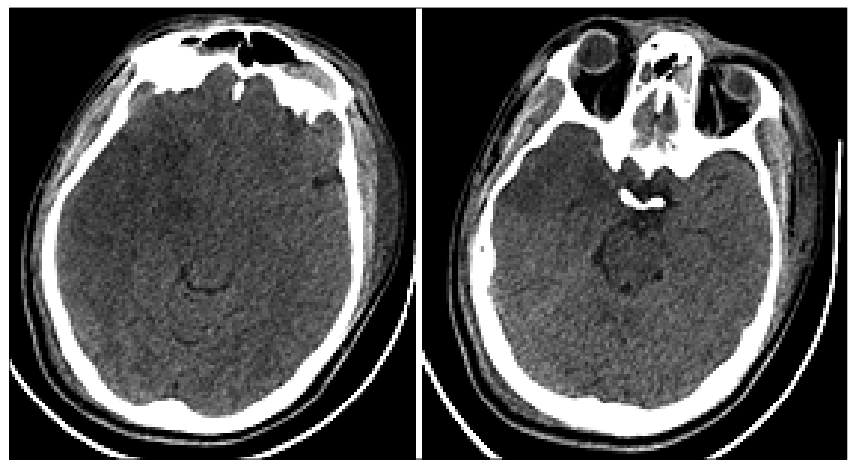

Figura 2. Tomografía axial computarizada simple de cráneo de base a convexidad a las 24 horas de evolución.

f) Cuidados intensivos. Ingresa a unidad de cuidados intensivos.

\section{4.- Evolución (24 horas de inicio).}

a) Imagenológica. Pérdida de relación sustancia gris sustancia blanca con pérdida de surcos, con zona de hipodensidad en territorio de arteria cerebral media derecha, desviación de la línea media de $5 \mathrm{~mm}$ con obliteración de ventrículo lateral derecho y desplazamiento hacia contralateral, cisternas perimescencefálicas parcialmente cerradas, tallo cerebral sin cambios de densidad (Figura 2).

b) Manejo General. Se suspende tratamiento antiplaquetario.

c) Manejo Quirúrgico. Craniectomía descompresiva.

\section{5.- Evolución (48 horas del inicio).}

a) Neurológica. Paciente se mantiene bajo sedación con intubación orotraqueal, anisocoria dependiente de pupila derecha $2 \mathrm{~mm}$.

b) Imagenológica. TAC de cráneo, se observa hernia transcalvaria frontotemporal derecha con recuperación de la línea media con obliteración de ventrículo lateral ipsilateral, establecimiento de infarto de territorio vascular perteneciente a la arteria cerebral media derecha (Figura 3).

\section{6.- Evolución (4 días de inicio).}

a) Neurológica. Extubación orotraqueal exitosa, movilización

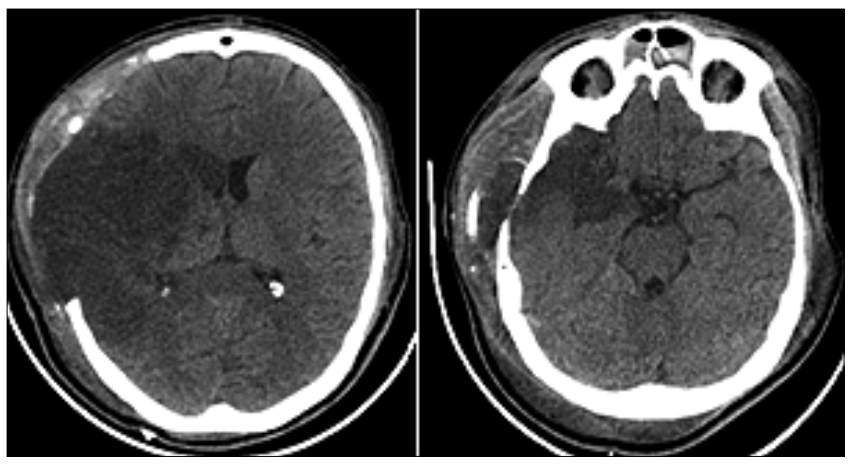

Figura 3. Tomografía axial computarizada simple de cráneo a las 108 horas de evolución.

de las cuatro extremidades, hemicuerpo derecho fuerza $4 / 5$, hemicuerpo izquierdo fuerza 2/5 con tendencia hacia la mejoría, función cognitiva con desorientación en espacio y tiempo, ptosis y miosis en globo ocular derecho con disminución de la agudeza visual ipsilateral.

b) Imagenológica. TAC de cráneo con zona de infarto cerebral, disminución de edema parenquimatoso (Figura 3).

7.- Evolución (7 días de inicio). Egreso hospitalario con tratamiento antiplaquetario por 6 meses y rehabilitación física.

\section{Discusión}

La disección traumática de la arteria carótida interna (DTACI) es una entidad poco frecuente y poco diagnosticada; la incidencia se describe entre 0,08 a 0,4\% $\%^{4}$.

En las contusiones cerradas de cuello la dinámica del traumatismo como hiperextensión o rotación forzada del cuello, debe orientar a la sospecha diagnóstica.

En el caso que se presenta, la progresión clínica transita de un estado íntegro, con estudios iniciales de imagen sin relevancia, hacia un deterioro clínico rápidamente progresivo, en menos de 12 horas, con presencia de tumefacción en cuello y dolor cervical que orientan al diagnóstico, aunado a ello, la información obtenida por la angiotomografía. 
Aproximadamente, el $70 \%$ de los pacientes son diagnosticados al presentar déficit neurológico importante, y el déficit se presenta en el $43 \%$ posterior al ingreso hospitalario, como la presentación clínica en este caso?

La evolución poco favorable mostrando delimitación gradual del territorio vascular de la arteria cerebral media por embolismo proveniente de la disección carotidea ipsilateral, llevó al paciente a un evento vascular cerebral isquémico severo que comprometió la vida y requirió de manejo quirúrgico de urgencia, para disminuir el aumento de la presión intracraneal.

La disección arterial puede causar eventos isquémicos ya sea por formación tromboembólica o por insuficiencia hemodinámica dada la severa estenosis u oclusión; como en el caso que se presenta en quien se evidenció disección carotidea y embolismo en la arteria cerebral media derecha ${ }^{4,19}$.

Las complicaciones secundarias al infarto cerebral deben tenerse en consideración dentro del espectro de la disección carotidea.

La tasa de mortalidad en esta patología es del $30 \%$, y las lesiones neurológicas secuelares $80 \%^{2}$; constituye el $20 \%$ de los casos de infarto cerebral en adultos jóvenes ${ }^{7}$.

Esta patología amerita el conocimiento del tema, la identificación temprana y la toma de decisiones oportunas, por parte del médico, en quien denota el conocimiento de la enfermedad y fisiopatología

"Los ojos ven lo que la mente conoce".

\section{Conclusión}

En el traumatismo de alto impacto sobre la región cervical, con deterioro neurológico rápidamente progresivo, se debe plantear la disección traumática de la arteria carótida interna (DTACl), así como las complicaciones propias de la enfermedad.

El embolismo proveniente de la disección carotidea es una entidad poco frecuente pero con resultados devastadores para la función cerebral del paciente, es necesaria alta sospecha diagnóstica para toma oportuna de decisiones como lo fue en el caso presentado.

\section{Referencias}

1. Arauz A, Hoyos L, Espinoza C, Cantú C, Barinagarrementeria F, Román G. Dissection of cervical arteries: Long-termfollow-up study of 130 consecutive cases. Cerebrovasc Dis, 2006; 22:1504. https://doi.org/10.1159/000093244

2. Beletsky V, Nadareishvili Z, Lynch J, Shuaib A, Woolfenden A, Norris JW. Cervical arterial dissection: time for a therapeutic trial? Stroke, 2003; 34(12):2856-60. https://doi.org/10.1161/01. STR.0000098649.39767.BC

3. Leys D, Bandu L, Henon H, Lucas C, Mounier-Vehier F, Rondepierre $\mathrm{P}$, Godefroy $\mathrm{O}$. Clinical outcome in 287 consecutive young adults (15-45 years) with ischemic stroke. Neurology, 2002; 59(1):26-33. https://doi.org/10.1212/WNL.59.1.26

4. Lee V, Brown RD, Mandrekar JN, Mokri B. Incidence and outcome of cervical artery dissection. A population-based study. Neurology, 2006; 67(10):1809-12. https://doi.org/10.1212/01. wnl.0000244486.30455.71

5. Grotta JC, Albers GW, Broderick JP. Stroke Pathophysiology, Diagnosis, and Management, Sixth Edition, Elsevier, 2016. China.

6. Dharmasaroja P, Dharmasaroja P. Sports-related internal carotid artery dissection: Pathogenesis and therapeutic point of view. Neurologist, 2008: 14(5):307-311. https://doi.org/10.1097/ NRL.0b013e31816d65cd

7. Fabian TC, Patton JH, Croce MA, Minard G, Kudsk KA, Pritchard E. Blunt carotid injury: Importance of early diagnosis and anticoagulant therapy. Ann Surg, 1996; 223(5):513-25. https://doi. org/10.1097/00000658-199605000-00007

8. Sikkema T, Uyttenboogaart M, Eshghi O, De Keyser J, BrounsR, van DijkJM,et al. Intracranial artery dissection. Eur J Neurol, 2014; 21(6):820-6. http://doi.org/10.1111/ene.12384

9. Redekop GJ. Extracranial carotid and vertebral artery dissection: A review. Can J Neurol Sci, 2008; 35(2):146-52. https://doi. org/10.1017/s0317167100008556

10. Patel RR, Adam R, Maldjian C, Lincoln CM, Yuen A, Arneja A. Cervical carotid artery dissection: current review of diagnosis and treatment. Cardiol Rev, 2012; 20(3):145-52. http://doi. org/10.1097/CRD.0b013e318247cd15

11. Yang ST., Huang YC.,Chuang CC., Hsu PW. Traumatic internal carotid artery dissection. J Clin Neurosci, 2006; 13(1):123-128. http://doi.org/10.1016/j.jocn.2005.02.016

12. Cogbill TH, Moore EE, Meissner M, Fischer RP, Hoyt DB, Morris JA, et al. The spectrum of blunt injury to the carotid artery: A multicenter perspective. J Trauma, 1994; 37(3):473-9. https:// doi.org/10.1097/00005373-199409000-00024

13. Roldán-Valadez E, Corona-Cedillo R, Ruiz-González D, Del Valle R, Herrera-Serrano A, Sánchez-Sánchez JM. Disección traumática de la arteria carótida interna extracraneal con infarto en el territorio de la arteria cerebral media: Diagnóstico por imagen. Gac. Méd. Méx, 2006; 142(5):419-422 http://www.scielo.org.mx/scielo. php?script=sci_arttext\&pid=S0016-38132006000500011\&lng=es

14. Rahme R, Aoun S, McClendon J, Bendok B.Spontaneous cervical and cerebral arterial dissectionsdiagnosis and management. https://doi.org/10.1016/..nic.2013.03.013

15. López-Sánchez M, Ballesteros-Sanz MA, Pérez-Ceballos A, González-Fernández C, López-Espadas F. Disección traumática de la arteria carótida interna por el cinturón de seguridad: descripción de dos casos. Medicina Intensiva, 2009; 33(7):311-362. htto://doi.org/10.1016/j.medin.2008.12.001

16. Biffl WL, Moore EE, Offner PJ, Brega KE, Franciosen RJ, Burch JM. Blunt carotid arterial injuries: Implicartions of a new grading scale. J Trauma, 1994; 47(5):845-53. https://doi. org/10.1097/000053473-199911000-00004

17. Gonzáles Trujillo F, Moreno Mosquera YF, Sánchez Caro EM, Holguín Díaz Jl. Disección de vasos arteriales cráneo cervicales. Reporte de casos presentados durante 2 años en una institución clínica de Colombia. Discusión con énfasis en tratamiento. Acta Neurol Colomb, 2018; 34(1):85-91. https://doi. org/10.22379/24224022183

18. Desfontaines $P$, Despland A. Dissectionof the internal carotid artery: aetiology, symptomatology, clinical and neurosonological follow-up and treatment in 60 consecutive cases. Act Neurol Psych Belg, 1995; 95(4):226-34.

19. Kenneth P, Madden K, Percy N, Karanjia, Adams H, Clarke W. Accuracy of initial stroke subtype diagnosis in the TOAST study. Neurology, 1995; 45 (11): 1975-79. https://doi.org/10.1212/ wnl.45.11.1975 